\title{
Periodic High-Conductance States in Spinal Neurons during Scratch-Like Network Activity in Adult Turtles
}

\author{
A. Alaburda, ${ }^{1}$ R. Russo, ${ }^{2}$ N. MacAulay, ${ }^{3}$ and J. Hounsgaard ${ }^{3}$ \\ ${ }^{1}$ Department of Biochemistry and Biophysics, Faculty of Natural Sciences, Vilnius University, 03101 Vilnius, Lithuania, ${ }^{2}$ Unidad Asociada Neurofisiologia, \\ Instituto de Investigacious Biologicas Clemente, Estable, Facultad de Ciencias, CP-11600 Montevideo, Uruguay, and ${ }^{3}$ Department of Medical Physiology, \\ Panum Institute, University of Copenhagen, DK-2200 Copenhagen, Denmark
}

Intense synaptic activity may alter the response properties of neurons in highly interconnected networks. Here we investigate whether the excitability and the intrinsic response properties of neurons in the spinal cord are affected by the increased synaptic conductance during functional network activity. Scratch episodes were induced by mechanical stimulation in the isolated carapace-spinal cord preparation from the adult turtle. Intracellular recordings revealed a dramatic increase in synaptic activity in interneurons and motoneurons during scratch activity. Superimposed slow depolarizing waves were phase-related to the rhythmic bouts of spike activity in the hip flexor nerve. The increase in synaptic conductance in interneurons and motoneurons varied with the scratch rhythm. During individual episodes, the conductance shifted smoothly with the scratch rhythm from near-resting levels to levels two to four times higher. In slice experiments, we found that even moderate increases in the conductance of motoneurons suppressed the slow afterhyperpolarization and the plateau potentials. We conclude that the excitability and the intrinsic response properties of spinal neurons are periodically quenched by high synaptic conductance during functional network activity.

Key words: spinal cord integrated preparation; modulation; membrane conductance; motoneuron; reflex; synaptic plasticity

\section{Introduction}

The intrinsic response properties provided by voltage-gated ion channels play an important role in the shaping of neuronal firing patterns (Llinas, 1988). This role, however, is moderated by synaptic conductance during network activity. The additional conductance induced by synaptic activity is related to the number of simultaneously active neurons, their firing frequencies, the synaptic connectivity, and the strength of synaptic connections. Consequently, the contribution of intrinsic response properties of highly interconnected neurons in large-scale networks, such as the cerebral cortex, is minimal during intense activity (Destexhe et al., 2003), but it plays a crucial functional role in oligocellular networks such as the stomatogastric ganglion (Nusbaum and Beenhakker, 2002). It is not clear whether and when highconductance states are obligatory ingredients of functional activity in large-scale networks. Sparsely coded functional activity in the cerebral cortex (Brecht et al., 2004) provides strong evidence for low-conductance states in which the intrinsic response properties would have a prominent role. The aim of the present study was therefore to investigate the conductance state in neurons during functional activity in a large-scale network in which neuronal response properties are thought to play a prominent role.

Received March 3, 2005; revised May 19, 2005; accepted May 22, 2005.

This work was supported by the Lundbeck Foundation, the European Union, the Statens Sundhedsvidenskabelige Forskniugràd, the Lithuanian State Science and Studies Foundation, and a NATO reintegration grant.

Correspondence should be addressed to J. Hounsgaard, Department of Medical Physiology, Panum Institute, University of Copenhagen, Blegdamsvej3,DK-2200 Copenhagen North, Denmark.E-mail:j.hounsgaard@mfi.ku.dk. DOI:10.1523/JNEUROSCI.0843-05.2005

Copyright $\odot 2005$ Society for Neuroscience $\quad$ 0270-6474/05/256316-06\$15.00/0
We used the isolated carapace-spinal cord preparation from turtles. In this preparation, scratch-like network activity induced by a sensory stimulus (Keifer and Stein, 1983) can be combined with stable intracellular recording in voltage-clamp and currentclamp modes (Alaburda and Hounsgaard, 2003). Scratching is produced by a large-scale network involving several spinal segments (Mortin and Stein, 1989; Stein, 2005). Motoneurons and interneurons in these segments express cell type-specific response properties (Russo and Hounsgaard, 1999) modulated by synaptic activity (Perrier et al., 2002; Alaburda and Hounsgaard, 2003). At the same time, scratch episodes in the spinal turtle in vivo are associated with a considerable conductance increase in motoneurons (Robertson and Stein, 1988). In the present study, we find that the intrinsic response properties of motoneurons and interneurons are quenched by increased synaptic conductance during rhythmic scratch network activity.

\section{Materials and Methods}

Integrated preparation. Red-eared turtles (Chrysemys scripta elegans; $n=$ 16) placed on crushed ice $2 \mathrm{~h}$ before surgery to induce hypothermic anesthesia (Melby and Altman, 1974) were killed by decapitation. Blood was substituted by perfusion with a Ringer's solution containing the following (in mM): $120 \mathrm{NaCl}, 5 \mathrm{KCl}, 15 \mathrm{NaHCO}_{3}, 2 \mathrm{MgCl}_{2}, 3 \mathrm{CaCl}_{2}$, and 20 glucose, saturated with $98 \% \mathrm{O}_{2}$ and $2 \% \mathrm{CO}_{2}$ to obtain $\mathrm{pH}$ 7.6. Transverse cuts were made to isolate the carapace containing the D4-D10 spinal cord segments (Alaburda and Hounsgaard, 2003). The hip flexor nerves puboischiofemoralis internus and pars anteroventralis that innervate the muscle were exposed and cut. The spinal cord was transected at the D10 vertebral segment. The vertebral laminas and spinal cord were removed 3-5 $\mathrm{mm}$ from the rostral end of the preparation. The caudal cut end of the carapace was glued to a Plexiglas platform and mounted in a 
holder. A small Plexiglas slab was glued to the carapace at the rostral cut edge and a plastic tube for perfusion of the spinal canal was installed.

Slice preparation. Transverse slices ( $1.5-2 \mathrm{~mm}$ thick) were obtained from the lumbar enlargement of adult turtles (C. scripta elegans; $n=12$ ) anesthetized by intraperitoneal injection of $100 \mathrm{mg}$ of sodium pentobarbitone and killed by decapitation.

All experiments were performed at room temperature $\left(20-22^{\circ} \mathrm{C}\right)$ in Ringer's solution. In slice experiments, ionotropic glutamate receptors were blocked by $25 \mu \mathrm{m}$ 6-cyano-7-nitroquinoxaline-2,3-dione (CNQX) and $50 \mu \mathrm{M}$ DL-2-amino-5-phosphonopentanoic acid (DL-AP-5) added to the Ringer's solution.

The surgical procedures used for the integrated preparation and the slice preparation complied with Danish legislation and were approved by the controlling body under the Ministry of Justice.

Recordings. Intracellular recordings in current-clamp and voltageclamp modes were performed with an Axoclamp-2A amplifier (Axon Instruments, Union City, CA). Glass pipettes were filled with a mixture of $0.9 \mathrm{~m}$ potassium acetate and $0.1 \mathrm{M} \mathrm{KCl}$. Voltage-clamp recordings were performed in the discontinuous service mode at a sample rate of 5-8 $\mathrm{kHz}$, with a gain of $0.7-1.5 \mathrm{nA} / \mathrm{mV}$ and a low-pass filter of $0.1 \mathrm{kHz}$. In some voltage-clamp experiments, action potentials were eliminated by 2-(triethylamino)- $N$-(2,6-dimethylphenyl) acetamide (QX-314; Sigma, St. Louis, MO) added to the pipette solutions ( $0.2 \mathrm{M}$ final concentration). In the integrated preparation, intracellular recordings were obtained from neurons in segment D10. The database consists of intracellular recordings from 24 motoneurons obtained in the motor nucleus in the ventral horn and 25 interneurons recorded in the intermediary zone and the deep dorsal horn.

Recordings were accepted if neurons had a stable membrane potential more negative than $-50 \mathrm{mV}$. Data were sampled at $20 \mathrm{kHz}$ with a 12-bit analog-to-digital converter (Digidata 1200; Axon Instruments), displayed by means of Axoscope and Clampex software (Axon Instruments), and stored on a hard disk for later analysis.

In the integrated preparation, hip flexor nerve activity was recorded with a differential amplifier (Iso-DAM8; World Precision Instruments, Sarasota, FL) using a suction pipette. The bandwidth was $100 \mathrm{~Hz}$ to 1 $\mathrm{kHz}$.

Stimulation. Mechanical stimulation was performed with the firepolished tip of a bent glass rod mounted to the membrane of a loudspeaker controlled with a function generator (Rosenberg, 1986). Stimuli were applied ventrally in carapace marginal shields M8-M9, which are the receptive fields for pocket scratching and the transition zone between the receptive fields for pocket and rostral scratching (Mortin et al., 1985).

Data analysis. In current-clamp mode, the amplitude of the slow spike afterhyperpolarization (AHP) was measured $30 \mathrm{~ms}$ after the peak of the action potential with the peak of the fast afterhyperpolarization as a reference. The conductance of neurons was measured from the voltage response to $150 \mathrm{~ms}$ hyperpolarizing current pulses applied at $0.5 \mathrm{~Hz}$. To reduce the influence of fast and slow fluctuations in membrane potential, a reference potential was obtained by linear interpolation of the average membrane potential 70-20 ms before the pulse and the average membrane potential $20-70 \mathrm{~ms}$ after the pulse. The average membrane potential $80-130 \mathrm{~ms}$ from the onset of the pulse was taken as the test potential. The estimated conductance was then obtained by dividing the amplitude of the current injected during the pulse with the difference between the reference potential and the test potential. Double-referent phase analysis (Stein and Daniels-McQueen, 2002) was performed on neurons that fired more than two action potentials during the depolarizing waves of scratch-like network activity.

In voltage-clamp mode, the conductance was derived from current traces from at least three holding potentials [adopted from Borg-Graham et al. (1998)]. The traces for analysis were aligned in time with the two first bouts in nerve activity. Data were analyzed statistically by using a two-populations (paired) $t$ test (Origin software; Microcal Software, Northampton, MA). Significance was accepted at $p<0.05$. If not otherwise specified, data are presented as means \pm SEM (this is for doublereferent data).

Drugs. In slice experiments, the following transmitter receptor agonists and antagonists were bath-applied: CNQX (25 mM) and DL-AP-5
(50 mM) from Tocris Cookson (Bristol, UK), and glycine (300-400 $\mu \mathrm{M}$ ), muscimol hydrobromide $(0.5-1.5 \mu \mathrm{M})$, and $( \pm)-1-[2,5]$-dimethoxy-4iodophenyl-2-aminopropane (DOI; $50 \mu \mathrm{M}$ ) from Sigma. QX-314 (0.2 M) was added to the pipette solution to block action potentials.

\section{Results}

Figure 1 illustrates the typical pattern of changes in synaptic activity (Fig. $1 A$ ) and input resistance (Fig. 1C) in current-clamp recordings from spinal neurons during a scratch episode. Less than $500 \mathrm{~ms}$ from the onset of a mechanical stimulus in the receptive field on the carapace, the synaptic activity increased dramatically and resulted in rapid fluctuations in membrane potential, as illustrated in Figure $1 A$, insets. Superimposed on the rapidly fluctuating membrane potential, slow waves of depolarization reached the spike threshold in 29 of 41 cells (13 of 18 motoneurons and 16 of 23 interneurons). In each cell, these waves had a characteristic phase relationship to the periodic bouts of activity recorded in the ipsilateral hip flexor nerve (Fig. $1 A$ ). We calculated the double-referent phase to describe the start and end phases of neuron firing relative to hip flexor nerve activity (Stein and Daniels-McQueen, 2002). In 11 of 13 motoneurons, the start and end phase of activity was within the ON phase of the hip flexor nerve (Fig. $1 B$ ). Some of these motoneurons, especially with later start phase, may be the knee extensor motoneurons, active during rostral scratching (Robertson and Stein, 1988). In interneurons $(n=9)$, the start and end phase relationship ranged from phase-advanced neurons to phase-delayed neurons with respect to hip flexor nerve activity (Fig. $1 B$ ).

The irregular firing frequency observed in motoneurons and interneurons during scratch episodes may be a simple consequence of the fluctuations in membrane potential but might also be aided by an increase in neuron conductance caused by the increased synaptic activity. We therefore estimated the change in neuron conductance during scratch episodes from the voltage change induced by hyperpolarizing current pulses, as illustrated in Figure $1 C$. Not surprisingly, the average neuron conductance was significantly increased during scratching in all cells tested $(68 \pm 11 \% ; n=49$; motoneurons, $57 \pm 10 \%, n=24$; interneurons, $79 \pm 21 \%, n=25)$. In addition, however, a cyclic variation in estimated input resistance was noticed during scratch episodes (Fig. 1C). The peak conductance increased by $280 \pm 55 \%(n=$ 49) (motoneurons, $262 \pm 66 \%, n=24$; interneurons, $297 \pm$ $90 \%, n=25$ ). As qualitatively noticed in vivo (Robertson and Stein, 1988), substantial increases in conductance were not always accompanied by significant changes in membrane potential (Fig. 1C, arrows).

One source of variability in estimates of input resistance in current clamp during scratch episodes is the fast and slow fluctuations in membrane potential. To eliminate this factor and improve the time resolution, the method introduced by BorgGraham et al. (1998) was adopted to derive the conductance changes from voltage-clamp records during scratch episodes (Fig. 2A). The current generated during scratch activity was recorded during at least three consecutive episodes, each at a different holding potential. In experiments in which the recording electrode did not contain QX-314, holding potentials were kept at levels more hyperpolarized than the spike threshold ( -55 to $-130 \mathrm{mV}$ ). In this voltage range, turtle spinal neurons display approximately linear current-voltage relationships at rest (Russo and Hounsgaard, 1996a,b; Delgado-Lezama et al., 1997; Svirskis and Hounsgaard, 1998). During scratch episodes, the conductance was derived from the linear relationship between the average current in $100 \mathrm{~ms}$ time segments at three different holding 
potentials (see Materials and Methods). In 35 of 36 neurons tested (27 of 27 motoneurons and 8 of 9 interneurons), the conductance during scratch episodes changed rhythmically with the bouts of hip flexor nerve activity from values near resting conductance and reached peak values up to 4 times higher. The mean and maximum conductance during scratching increased by $35 \pm 8$ and $79 \pm 13 \%(n=36)$ (motoneurons, $38 \pm 11$ and $84 \pm 17 \%$, $n=27$; interneurons, $24 \pm 7$ and $61 \pm$ $12 \%, n=9)$.

In a few experiments, individual inhibitory synaptic events could be discerned during network activity. In the voltageclamp experiment in Figure $2 A$, we noticed that when the membrane potential was held at $-35 \mathrm{mV}$, prominent transient outward currents occurred during the slow waves of net inward current as well as during the intervening periods of net outward current. These transients were reduced in amplitude at $-55 \mathrm{mV}$ and absent at $-75 \mathrm{mV}$, as highlighted in the insets. Similarly, in the few cells in which individual inhibitory synaptic potentials could unambiguously be resolved in current clamp, the frequency of inhibitory synaptic potentials peaked during the depolarizing waves of scratch episodes (Fig. $2 B$ ).

In theory (Bernander et al., 1991; Rapp et al., 1992; Shadlen and Newsome, 1994) and in experiments in neocortical neurons (Destexhe and Pare, 1999; Destexhe et al., 2003), synaptically induced fluctuations in membrane potential and conductance of the order of magnitude observed here have dramatic effects on active and passive postsynaptic integration. Spike afterhyperpolarizations and plateau potentials are prominent and well investigated intrinsic response properties in turtle motoneurons (Hounsgaard et al., 1988; Russo and Hounsgaard, 1999; Alaburda et al., 2002; Stauffer et al., 2005). We investigated the sensitivity of these properties to pharmacologically increased conductance in motoneurons in transverse slices of the turtle spinal cord. Figure $3 A$ shows the response to depolarizing current pulses before and after application of $1.5 \mu \mathrm{M}$ muscimol, a selective $\mathrm{GABA}_{\mathrm{A}}$ receptor agonist. Muscimol increased the membrane conductance by $80 \%$. This increased the threshold for spike generation and reduced the slow afterhyperpolarization. In all 15 motoneurons investigated, the slow afterhyperpolarization $30 \mathrm{~ms}$ after action potentials was significantly reduced in amplitude or abolished by the increased membrane conductance $(35-150 \%)$ induced by bath-applied muscimol $(0.5-1.5 \mu \mathrm{M} ; p<0.0003 ; n=10)$ or glycine $(300-400$ $\mu \mathrm{M} ; p<0.003 ; n=5)$. In turtle motoneurons, the generation of

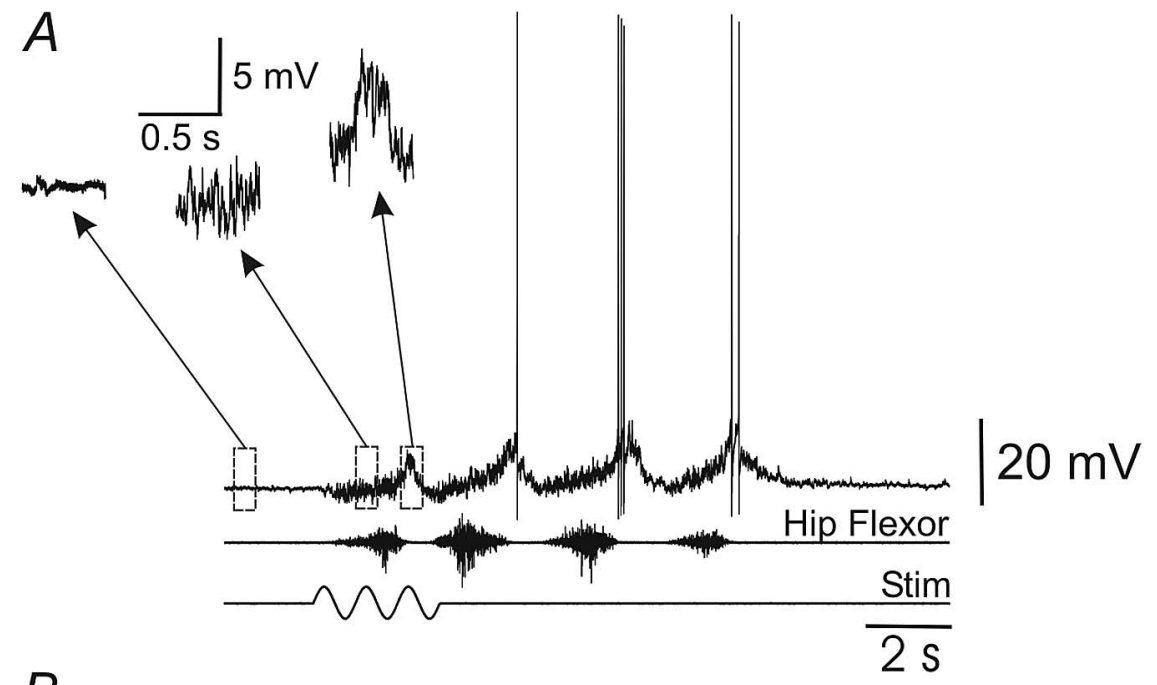

Motoneurons

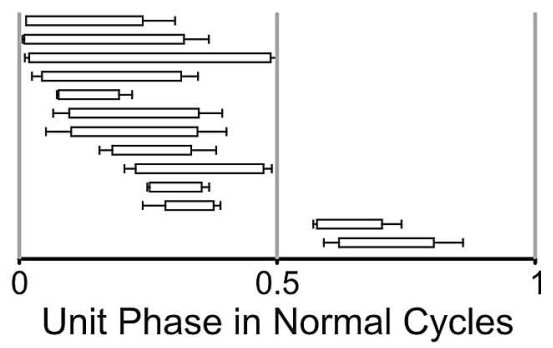

Interneurons
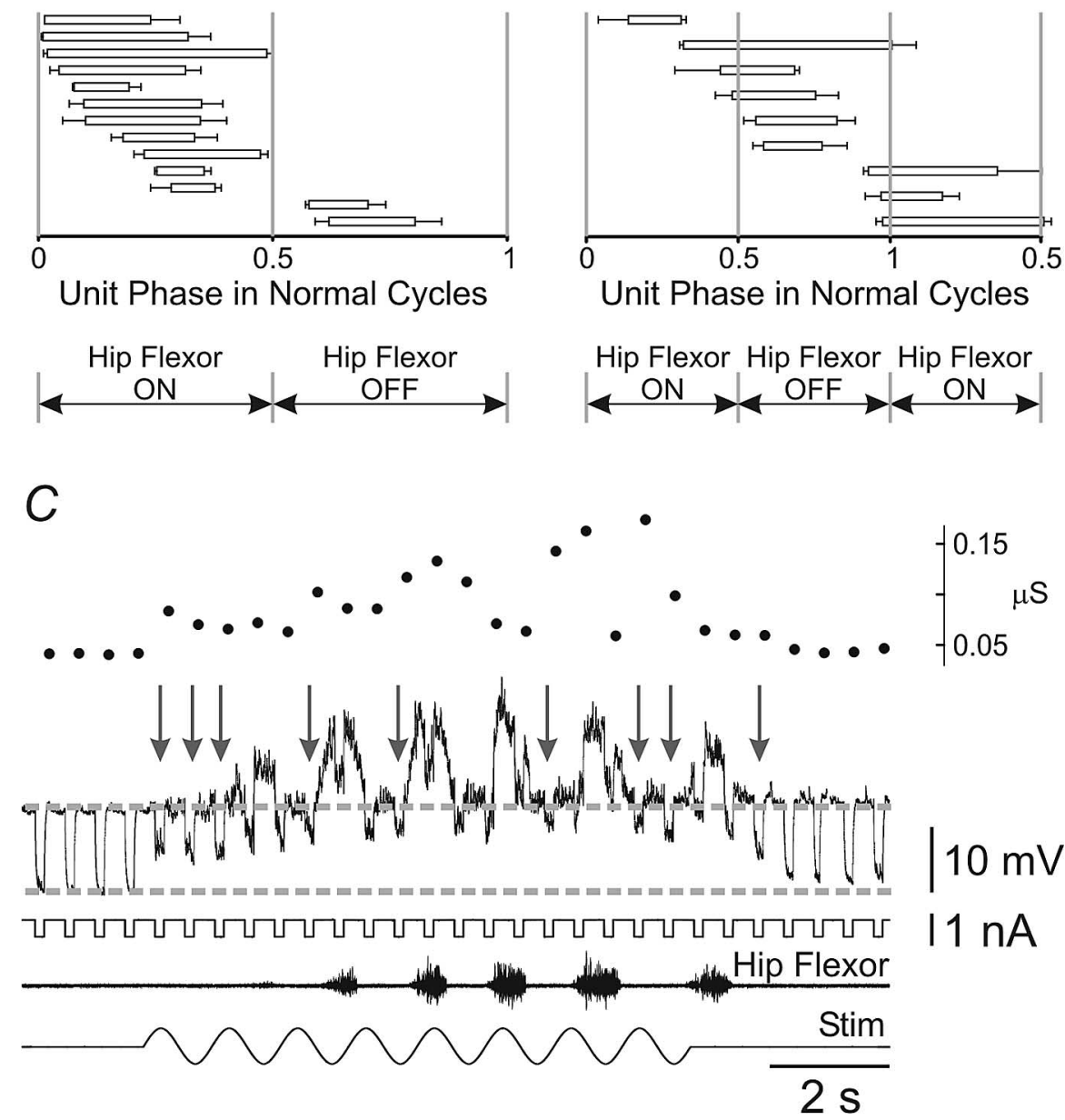

Figure 1. Synaptic activity and conductance increase during scratching. $\boldsymbol{A}$, Increased synaptic activity during scratching, highlighted in insets. B, Double-referent mean ON and OFF phases (with and without angular deviation) of motoneurons (left) and interneurons (right) recorded. C, Increased conductance during scratching. Arrows mark decreased responses to hyperpolarizing current pulses near the resting membrane potential. $\boldsymbol{A}-\boldsymbol{C}$ are recordings from three different motoneurons. Stim, Stimulation.

plateau potentials during depolarization is a latent property facilitated by activation of metabotropic glutamate $1,5-\mathrm{HT}_{2}$, and muscarine receptors (Delgado-Lezama et al., 1997; Svirskis and Hounsgaard, 1998; Perrier and Hounsgaard, 2003). We found 

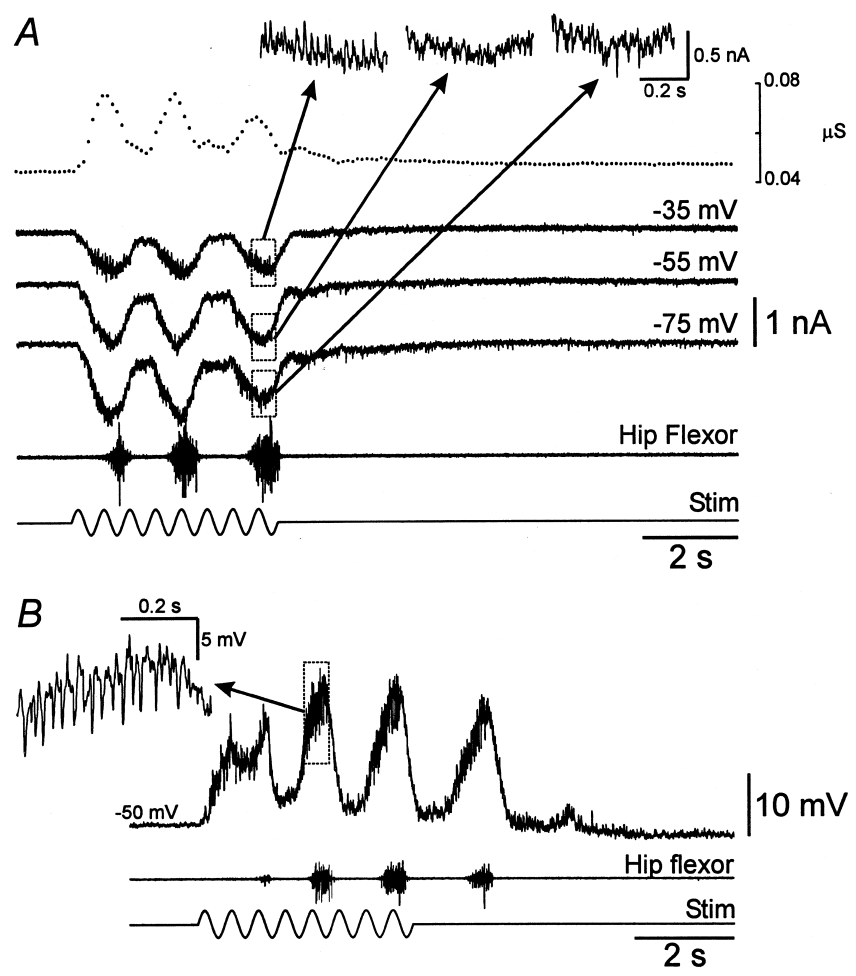

Figure 2. Cyclic variation in neuron conductance and inhibition during scratching. $A$, Neuron conductance calculated from current values at three different holding potentials (see Materials and Methods). Note the cyclic variation in conductance with bouts of nerve activity. Insets, Fragments of current traces in higher resolution. Fast outward current transients during a net inward current at $-35 \mathrm{mV}$ are reduced and disappear at more hyperpolarized levels. $\boldsymbol{B}$, Fast hyperpolarizing transients during a depolarizing wave in an interneuron during scratching. Stim, Stimulation.

$A$

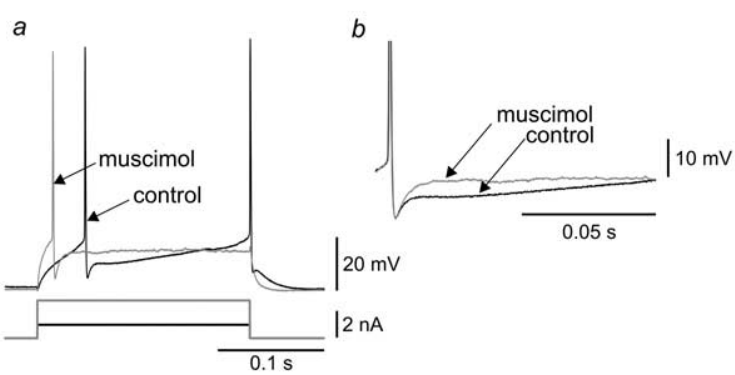

$B$

a

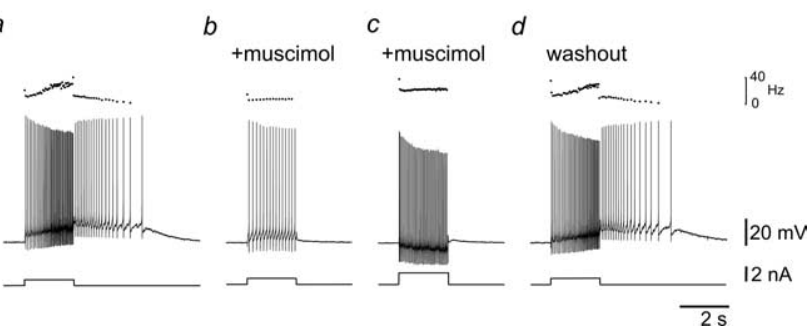

Figure 3. Slow AHPs and plateau potentials quenched by a muscimol-induced conductance increase. $\boldsymbol{A a}$, Response to a depolarizing current pulse in control (black) and in the presence of muscimol (gray). $\boldsymbol{A} \boldsymbol{b}$, Superimposed action potentials from $\boldsymbol{A} \boldsymbol{a}$. Note the decrease in amplitude of the slow AHP. $\boldsymbol{B}$, Plateau potentials eliminated by conductance increase. $\boldsymbol{B} \boldsymbol{a}$, Response to a depolarizing current pulse in a motoneuron in the presence of $\mathrm{DOl}$. Note accelerating firing and afterdischarge. $\boldsymbol{B} \boldsymbol{b}$, Absence of accelerating firing and afterdischarge after application of muscimol. $B \boldsymbol{C}$, Even larger depolarizing current pulses unable to induce acceleration and afterdischarge. $\boldsymbol{B d}$, Acceleration and afterdischarge reappeared after washout of muscimol.

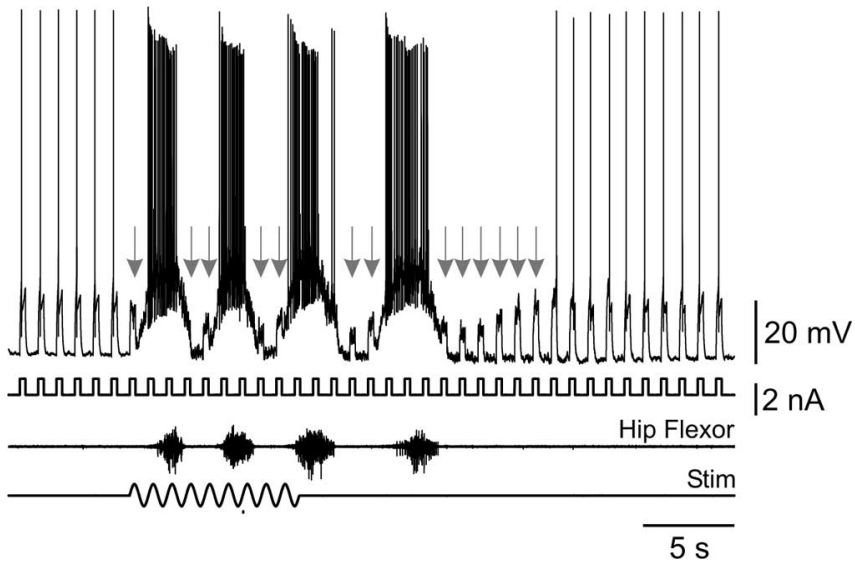

Figure 4. Excitability of spinal motoneurons reduced during scratching. The excitability was tested by injecting depolarizing current pulses adjusted to elicit one action potential at rest. Arrows mark action potential failures to stimuli (Stim) during scratching.

that the generation of plateau potentials in motoneurons was abolished by even a moderate increase in membrane conductance induced by application of muscimol or glycine. In the experiment illustrated in Figure $3 B$, plateau potentials were activated by depolarization in the presence of the $5-\mathrm{HT}_{2}$ agonist DOI $(50 \mu \mathrm{M})$ (Fig. $3 \mathrm{Ba}$ ). Application of $1 \mu \mathrm{M}$ muscimol led to a $22 \%$ increase in membrane conductance and abolished the generation of plateau potentials by the depolarizing pulse (Fig. $3 \mathrm{Bb}$ ). The plateau potentials were not restored by more intense depolarization (Fig. $3 B c$ ) but fully recovered after removal of muscimol (Fig. $3 B d$ ). The generation of plateau potentials facilitated by DOI $(50 \mu \mathrm{M})$ was abolished or severely inhibited by an increase in conductance of $10-100 \%$ in seven motoneurons tested with muscimol $(0.5$ $\mu \mathrm{M})$ and an increase in conductance of $20-60 \%$ in six motoneurons tested with glycine $(200 \mu \mathrm{M})$.

We noted that the threshold for action potentials in response to depolarizing current pulses increased when the membrane conductance of motoneurons was increased by application of glycine or muscimol. We therefore tested whether this effect was apparent during scratch episodes in the integrated preparation. As illustrated by the recording from the motoneuron shown in Figure 4, we found that the conductance increase during scratching reduced neuronal excitability. In this experiment, a $0.5 \mathrm{~s}$ depolarizing current pulse, adjusted in amplitude to evoke a single action potential at rest, was repeated at $0.5 \mathrm{~Hz}$. From the onset of scratching, the response to the current pulse was reduced in amplitude and did not evoke spikes outside the firing period during the depolarizing waves. In this cell, the intense synaptic activity and the conductance gradually declined during a $5 \mathrm{~s}$ after the last depolarizing wave. The decreased response to depolarizing current pulses during scratch episodes was observed in 11 of 14 motoneurons tested.

\section{Discussion}

In this study, we find a phasic increase in neuron conductance in motoneurons and interneurons during scratch-like network activity induced by sensory stimulation in the isolated lumbar carapace-spinal cord preparation from adult turtles. Our results suggest that high-conductance states are produced by a balanced increase in excitatory and inhibitory synaptic activity. During the high-conductance state, neuronal firing is determined by synaptically induced fluctuations in membrane potential rather than intrinsic response properties. In slice experiments, we demon- 
strate that even a moderate increase in motoneuron conductance reduces excitability and quenches the intrinsic response properties provided by slow afterhyperpolarizations and plateau potentials. In agreement, we find a periodic reduction in neuron excitability during scratch-like network activity.

The experimental conditions for estimating neuronal input conductance during network activity are not ideal. The large increase in synaptic activity introduces rapid fluctuations in membrane potential in voltage recordings and in current during voltage clamp. In addition, the response to injected current pulses during voltage recordings is superimposed on waves of depolarization produced by the network activity. The fidelity of the voltage clamp in the single-electrode switch mode is limited (Wilson and Goldner, 1975), and the degree of space clamp is modulated by the changes in electrotonic length of the dendrites induced by variation in synaptic activity. Without ignoring these reservations, however, the general agreement between the independent estimates of conductance from voltage and current recordings strongly supports the qualitative conclusion that periodic highconductance states occur in motoneurons and interneurons during scratch episodes.

Our present techniques do not allow a quantitative evaluation of the ratio between excitation and inhibition during network activity. The finding that high-conductance states occur with moderate changes in membrane potential (Fig. $2 B$ ) supports the idea that these states are produced in spinal neurons by a balanced increase in inhibition and excitation, as shown previously for cortical neurons (Destexhe et al., 2003; Shu et al., 2003). The implication is that the depolarizing waves during scratch network activity, often associated with a large increase in conductance, are produced by a general increase in synaptic activity and a change in balance between excitation and inhibition. This is supported by the intense inhibitory synaptic activity recorded during depolarizing waves (Fig. $2 B$ ) and fast outward current transients during net inward current (Fig. $2 \mathrm{~A}$ ). A variation in the level of overall synaptic activity, and therefore neuron conductance, during scratch episodes is compatible with the in vivo finding that structured patterns of excitation and inhibition occur in a cell typespecific manner during scratching (Robertson and Stein, 1988).

In analogy with neurons in invertebrate oligocellular networks, the intrinsic response properties of motoneurons and interneurons in spinal motor networks are thought to shape firing patterns during rhythmic motor activity (Kernell, 1999; Russo and Hounsgaard, 1999; Grillner, 2003). As in previous experiments (Alaburda and Hounsgaard, 2003), signs of bistability and plateau potentials were not observed in motoneurons during scratch episodes. In the present study, even prominent plateau properties displayed by interneurons at rest were quenched during scratching (data not shown) supposedly because of the increase in synaptic conductance. In support, slow afterhyperpolarizations and plateau properties were severely affected in motoneurons in slices by conductance increases smaller than observed during network activity in the carapace-spinal cord preparation.

The absence of plateau properties during depolarizing waves suggests that intrinsic response properties of motoneurons and interneurons play a less prominent role during scratching than expected. However, the large increase in inhibitory and excitatory synaptic activity, as reflected by the rapid fluctuations in membrane potential and the increase in neuron conductance, may provide mechanisms for gain control (Chance et al., 2002; Prescott and De Koninck, 2003) and multiplicative synaptic integration (Rudolph and Destexhe, 2003). Likewise, the weight of prox- imal and distal synapses will vary with the conductance because of the change in electrotonic structure. It remains to be seen whether spinal motor networks also support sparsely coded functional activity in which intrinsic response properties would come into play. Good candidates could be postural motor activity and initiation of network activity from rest.

A preceding paper reported that activation of metabotropic receptors enhanced the intrinsic response properties mediated by L-type $\mathrm{Ca}^{2+}$ channels for tens of seconds after scratch network activity (Alaburda and Hounsgaard, 2003). Here we have shown that the same intrinsic response properties are attenuated by increased conductance caused by activation of ionotropic receptors during network activity. In this way, the signaling properties of neurons may be changed by several parallel mechanisms on different time scales.

\section{References}

Alaburda A, Hounsgaard J (2003) Metabotropic modulation of motoneurons by scratch-like spinal network activity. J Neurosci 23:8625-8629.

Alaburda A, Perrier JF, Hounsgaard J (2002) Mechanisms causing plateau potentials in spinal motoneurones. Adv Exp Med Biol 508:219-226.

Bernander O, Douglas RJ, Martin KA, Koch C (1991) Synaptic background activity influences spatiotemporal integration in single pyramidal cells. Proc Natl Acad Sci USA 88:11569-11573.

Borg-Graham LJ, Monier C, Fregnac Y (1998) Visual input evokes transient and strong shunting inhibition in visual cortical neurons. Nature 393:369-373.

Brecht M, Schneider M, Sakmann B, Margrie TW (2004) Whisker movements evoked by stimulation of single pyramidal cells in rat motor cortex. Nature 427:704-710.

Chance FS, Abbott LF, Reyes AD (2002) Gain modulation from background synaptic input. Neuron 35:773-782.

Delgado-Lezama R, Perrier JF, Nedergaard S, Svirskis G, Hounsgaard J (1997) Metabotropic synaptic regulation of intrinsic response properties of turtle spinal motoneurones. J Physiol (Lond) 504:97-102.

Destexhe A, Pare D (1999) Impact of network activity on the integrative properties of neocortical pyramidal neurons in vivo. J Neurophysiol 81:1531-1547.

Destexhe A, Rudolph M, Pare D (2003) The high-conductance state of neocortical neurons in vivo. Nat Rev Neurosci 4:739-751.

Grillner S (2003) The motor infrastructure: from ion channels to neuronal networks. Nat Rev Neurosci 4:573-586.

Hounsgaard J, Kiehn O, Mintz I (1988) Response properties of motoneurones in a slice preparation of the turtle spinal cord. J Physiol (Lond) 398:575-589.

Keifer J, Stein PS (1983) In vitro motor program for the rostral scratch reflex generated by the turtle spinal cord. Brain Res 266:148-151.

Kernell D (1999) Repetitive impulse firing in motoneurons: facts and perspectives. Prog Brain Res 123:31-37.

Llinas RR (1988) The intrinsic electrophysiological properties of mammalian neurons: insights into central nervous system function. Science 242:1654-1664.

Melby ECJ, Altman NH (1974) Handbook of laboratory animal science. Cleveland: CRC.

Mortin LI, Stein PS (1989) Spinal cord segments containing key elements of the central pattern generators for three forms of scratch reflex in the turtle. J Neurosci 9:2285-2296.

Mortin LI, Keifer J, Stein PS (1985) Three forms of the scratch reflex in the spinal turtle: movement analyses. J Neurophysiol 53:1501-1516.

Nusbaum MP, Beenhakker MP (2002) A small-systems approach to motor pattern generation. Nature 417:343-350.

Perrier JF, Hounsgaard J (2003) 5-HT2 receptors promote plateau potentials in turtle spinal motoneurons by facilitating an L-type calcium current. J Neurophysiol 89:954-959.

Perrier JF, Alaburda A, Hounsgaard J (2002) Spinal plasticity mediated by postsynaptic L-type $\mathrm{Ca}^{2+}$ channels. Brain Res Brain Res Rev 40:223-229.

Prescott SA, De Koninck Y (2003) Gain control of firing rate by shunting 
inhibition: roles of synaptic noise and dendritic saturation. Proc Natl Acad Sci USA 100:2076-2081.

Rapp M, Yarom Y, Segev I (1992) The impact of parallel fiber background activity on the cable properties of the cerebellar Purkinje cells. Neural Comput 4:518-533.

Robertson GA, Stein PS (1988) Synaptic control of hindlimb motoneurones during three forms of the fictive scratch reflex in the turtle. J Physiol (Lond) 404:101-128.

Rosenberg ME (1986) Carapace and plastron sensitivity to touch and vibration in the tortoise (Testudo hermanni and T. graeca). J Zool 208:443-455.

Rudolph M, Destexhe A (2003) Characterization of subthreshold voltage fluctuations in neuronal membranes. Neural Comput 15:2577-2618.

Russo RE, Hounsgaard J (1996a) Plateau-generating neurones in the dorsal horn in an in vitro preparation of the turtle spinal cord. J Physiol (Lond) 493:39-54.

Russo RE, Hounsgaard J (1996b) Burst-generating neurones in the dorsal horn in an in vitro preparation of the turtle spinal cord. J Physiol (Lond) 493:55-66.
Russo RE, Hounsgaard J (1999) Dynamics of intrinsic electrophysiological properties in spinal cord neurones. Prog Biophys Mol Biol 72:329-365.

Shadlen MN, Newsome WT (1994) Noise, neural codes and cortical organization. Curr Opin Neurobiol 4:569-579.

Shu Y, Hasenstaub A, McCormick DA (2003) Turning on and off recurrent balanced cortical activity. Nature 423:288-293.

Stauffer EK, Stuart DG, McDonagh JC, Hornby TG, Reinking RM (2005) Afterhyperpolarization-firing rate relation of turtle spinal neurons. J Comp Physiol [A] 191:135-146.

Stein PS (2005) Neuronal control of turtle hindlimb motor rhythms. J Comp Physiol [A] 191:213-229.

Stein PS, Daniels-McQueen S (2002) Modular organization of turtle spinal interneurons during normal and deletion fictive rostral scratching. J Neurosci 22:6800-6809.

Svirskis G, Hounsgaard J (1998) Transmitter regulation of plateau properties in turtle motoneurons. J Neurophysiol 79:45-50.

Wilson WA, Goldner MM (1975) Voltage clamping with a single microelectrode. J Neurobiol 6:411-422. 